\title{
Optimal Design of Lifting Platform Balance Control Algorithm: Co-Simulation and Experiment
}

\author{
Wang Xin ${ }^{1}$, Xu Miao ${ }^{1}$, Li Weiwei ${ }^{1}$, Bogdan Miedzinski ${ }^{2}$, Wang Shumao ${ }^{1}$ \\ ${ }^{1}$ Beijing Key Laboratory of Optimized Design for Modern Agricultural Equipment, \\ College of Engineering, China Agricultural University (East Campus), \\ Beijing, China \\ ${ }^{2}$ Institute of Innovative Technologies (EMAG), \\ Leopolda 31, 40-189 Katowice, Poland \\ wangxin117@cau.edu.cn
}

\begin{abstract}
The paper deals with balance control problem of lifting platform, collecting detailed first-hand information on its operation in reality. Sums up main challenges to be faced under processing of balance control. Recommends creative and representative control algorithms, comprehensively analysed basing on target platform developed by authors with respective following verification by simulation and testing. Relevant study was performed under three following steps: mathematical modelling, control system design and system verification. Therefore, dynamic model and ADAMS model have been established first based on actual structure of lifting platform. Next, a suitable control algorithm was selected and parameters of target models were set up using ADAMS/MATLAB co-simulation. Finally, both simulation and test results have been verified and compared to show their coincidence. It was found that control algorithms recommended in this paper can effectively improve as stability, reliability as well as security of operation of the lifting platform and can solve as a result the balance control problem of lifting platform at some extent in practice.
\end{abstract}

Index Terms-ADAMS; balance control; co-simulation; lifting platform; MATLAB.

\section{INTRODUCTION}

In recent years, lifting platforms have been extensively used for military, industry and civilian purposes. Therefore, the study on its balance control is paid more and more attention. For example, J. Chao sets up an electro-pneumatic stabilized platform driven by pneumatic muscles, and adopts an adaptive robust levelling control strategy based on the coupled MIMO system dynamics to remain balance of platform [1]. In turn, J. Zhang establishes dynamic model of vehicle platform, and recommends a method to achieve excellent dynamic performance of platform levelling process by using repeated balance control strategy [2]. The balance control system for micro platform of non-probe near field optical microscope, which is based on multi-point real-time

Manuscript received 10 April, 2017; accepted 11 August, 2017.

This research was funded by grant (No. 51405492) program for The National Natural Science Fund of China, grant (No. D151100003715001) and (No. D161100003216002) Beijing Municipal Science and Technology Commission project of China. detection and fuzzy adaptive PID control algorithm is discussed by some researchers [3].

This paper presents the developed testing model of the mobile platform installed on a wheeled vehicle. For this purpose, its dynamic model has been derived and respective PID-rule-based control algorithm, which allows direct rotation around $\mathrm{X}$ and $\mathrm{Z}$ axes to compensate for errors of unbalance angle, has been recommended. Estimation of effectiveness of control algorithm was performed by means of ADAMS and MATLAB Simulink co-simulation. Finally, analysis of simulation and experiment results shows that, satisfactory performance can be achieved when combining the control algorithm with properly selected PID regulator parameters.

\section{MODEL CREATION}

\section{A. Lifting Platform Design}

In order to ensure smooth operation of the lifting platform, it must possess at least three degrees of freedom (DOF) [4]-[6], that is, motion along $\mathrm{Y}$ axis, rotation around $\mathrm{X}$ and $\mathrm{Z}$ axes [7]. The structure of lifting platform indicates that each rotating DOF is achieved by one electrical push rod and four revolute joints, which are fixed in the middle of rotating plane and at both ends of electrical push rod. The actual structure of prototype vehicle with lifting platform, which is used as a simulation and testing model in this paper, is shown in Fig. 1.

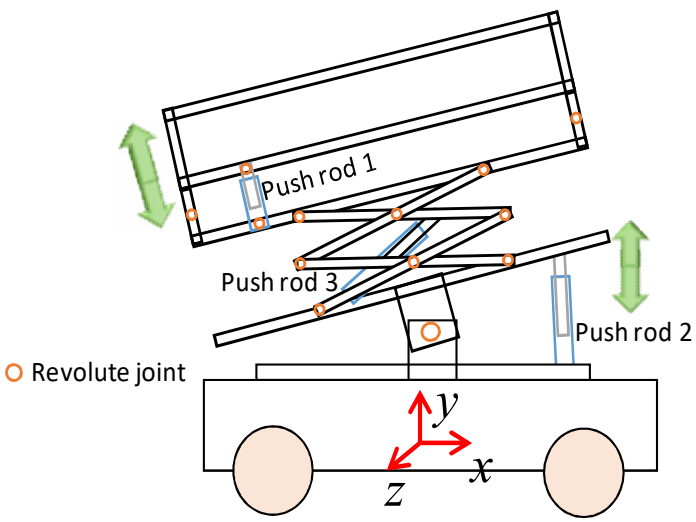

Fig. 1. Structure diagram of lifting platform. 


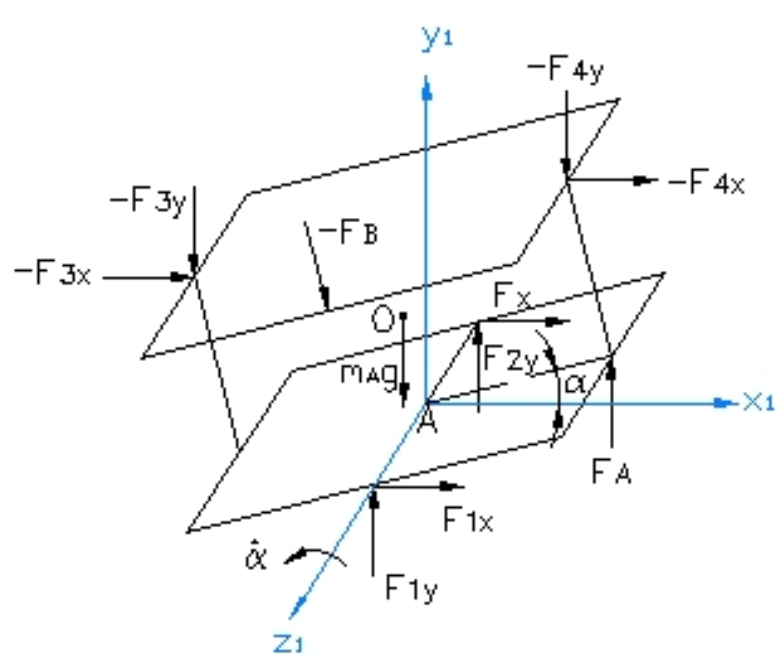

(a)

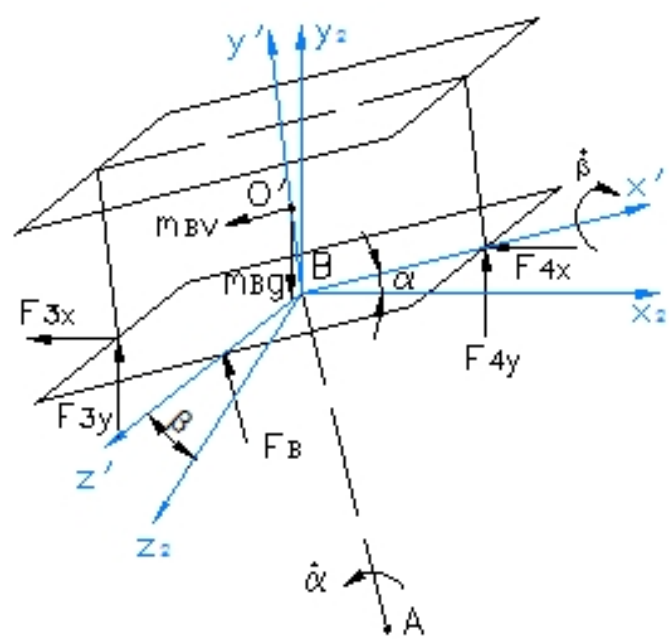

(b)

Fig. 2. Schematic structure of lifting platform: (a) - part A of lifting platform; (b) - part B of lifting platform.

\section{B. Dynamic Model}

A properly developed mathematical model enables system operation to be examined through experiments, without the need to build a physical system [4]. You can then perform various modifications of the mathematical model taking into account the influence of various input quantities and their values.

In first stage of study, the model of the developed lifting platform with rotation around $\mathrm{X}$ and $\mathrm{Z}$ axis has been established. To simplify the calculation, it was assumed that two push rods are located on a vertical plane and only subjected to axial direction force. The platform was then divided into two planes $\mathrm{A}$ and $\mathrm{B}$ with the centres of gravity $\mathrm{O}$ and O', which do not change location when lifting or levelling (Fig. 2).

Rectangular coordinate systems $\mathrm{O}_{1-\mathrm{X} 1 \mathrm{Y} 1 \mathrm{ZZ} 1}$ and $\mathrm{O}_{2-\mathrm{X} 2 \mathrm{Y} 2 \mathrm{Z} 2}$ were positioned in the middle of the Part A's and B's bottom, representing upper and lower part of lifting platform respectively. To simplify the calculation of dynamic model, the coordinate system of lifting platform $\mathrm{O}_{2-\mathrm{X}^{\prime} \mathrm{Y}^{\prime} \mathrm{Z}}$ ' has been referred to part $\mathrm{B}$, wherein, $\alpha$ is obliquity of $\mathrm{X}$ axis, $\beta$ is obliquity of $\mathrm{Z}$ axis, and $F_{A}, F_{B}$ are supporting forces of each push rod. As a result, the equation of motion of part $A$ can describe by the formula

$$
\begin{gathered}
J_{A} \dot{\alpha}=F_{A} \times \frac{L_{1}}{2} \times \cos \alpha+m_{A} \mathrm{~g} \times \\
\times \frac{h_{1}}{2} \times \tan \alpha-F_{3 y} \times \frac{L_{1}}{2} \times \cos \alpha+F_{4 y} \times \frac{L_{1}}{2} \times \cos \alpha+ \\
+F_{3 x}\left(h_{1} \times \cos \alpha-\frac{L_{1}}{2} \times \sin \alpha\right)+ \\
+F_{4 x}\left(h_{1} \times \cos \alpha+\frac{L_{1}}{2} \times \sin \alpha\right),
\end{gathered}
$$

where the part A is rotated only around $\mathrm{Z} 1$ axis, and $\dot{\alpha}$ is its rotational angular velocity.

Since, resultant force has been transmitted to $\mathrm{Z} 1$ axis, therefore, mechanical equilibrium equations are as follows:

$$
\begin{gathered}
F_{1 \mathrm{x}}+F_{2 \mathrm{x}}-F_{3 \mathrm{x}}-F_{4 \mathrm{x}}-F_{B} \times \sin \alpha=0, \\
\left(F_{1 \mathrm{x}}-F_{2 \mathrm{x}}-F_{B} \times \sin \alpha\right) \times \frac{L_{2}}{2}=0, \\
m_{A} \mathrm{~g}-F_{A}-F_{B} \times \cos \alpha-F_{1 y}-F_{2 y}-F_{3 y}-F_{4 y}=0, \\
\left(F_{2 y}-F_{1 y}-F_{B} \times \cos \alpha\right) \times \frac{L_{2}}{2}=0 .
\end{gathered}
$$

As regards the part $B$ it is allowed to rotate both around the $\mathrm{Z}$ and $\mathrm{X}^{\prime}$ axes. Whereas, $\dot{\beta}$ is the rotational angular velocity around $\mathrm{X}^{\prime}$ axis and $\beta$ is the angle between the bottom and horizontal planes.

The equation of rotational motion around $X^{\prime}$ axis has the form

$$
J_{B} \times \dot{\beta}=F_{B} \times \frac{L_{2}}{2} \times \cos \beta,
$$

whereas equation of rotation around $Z_{1}$ axis can be expressed as:

$$
\begin{gathered}
m_{B} \ddot{\alpha} \times\left(h+\frac{h_{1}}{2} \times \cos \alpha\right)= \\
=\left(F_{3 x}+F_{4 x}\right) \times \cos \alpha+m_{B} \mathrm{~g} \times \sin \alpha, \\
m_{B} \dot{\alpha}^{2} h=m_{B} g \cos \alpha-\left(F_{3 Y}-F_{4 Y}\right) \sin \alpha-F_{B} \cos \alpha,
\end{gathered}
$$

where $J_{A}$ is rotational inertia of part $\mathrm{A}$ around $\mathrm{Z}$ axis, $J_{B}$ is rotational inertia of part $\mathrm{B}$ around $\mathrm{X}^{\prime}$ axis, $m A$ and $m B$ are mass of part A and $\mathrm{B}, L_{1}$ and $L_{2}$ are length and width of platform respectively, $h 1$ is height of part $\mathrm{A}, h 2$ is height of part $\mathrm{B}$, and $h$ is distance between $\mathrm{Z}_{1}$ and $\mathrm{Z}_{2}$ axes.

\section{CONTROL STRATEGY}

Currently two control algorithms are used for balance control strategy: displacement error control algorithm (DEC) and angle error control algorithm (AEC) [8]-[10]. The DEC is used to make system adjustment, that is, fixes one point, and 
then detects and eliminates a position error between the fixed point and removal points. On the contrary, AEC is used to detect angle error and decrease it to 0 degree by controlling systematic movement. Taking into account the convenience of detection, authors applied the AEC algorithm. Flow chart of balance control of AEC [11] is shown in Fig. 3.

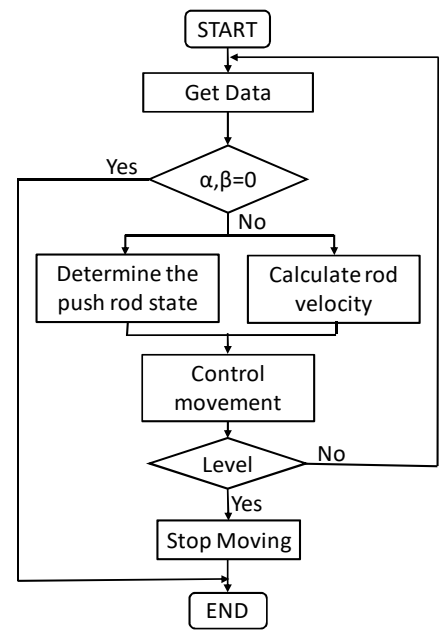

Fig. 3. Flow chart of balance control.

At first, angle information $(\alpha, \beta)$ is obtained from sensor and compared with ideal angle $\left(0^{\circ}\right)$. If $\alpha>0^{\circ}$, rod 1 needs to drop down to decrease angle. If $\alpha<0^{\circ}, \operatorname{rod} 1$ needs to rise up to increase angle. When $\alpha=0^{\circ}$, rod 1 will not adjust. Because the ideal angel $\left(\alpha=0^{\circ}\right)$ is not sustained in practice, it was necessary to set up an adjustable range to improve stability of the balance control.

\section{Simulation Method}

\section{A. ADAMS Model}

Under bump, slope and hollow road conditions, the obliquity and stability of the lifting platform are greatly affected by nonlinear characteristics of push-rods, lifting system. Therefore, model that can simply and precisely reproduce dynamic characteristics of these systems is needed. A. Sapietova indicates that ADAMS software is very effective and useful providing right mapping of independent driving characteristics of both push-rods [12].

Considering the analysis of the impact of actual parameters, the respective lifting platform model has been developed using ADAMS program. The simplified procedure can be described as follows:

1. Measure dimensions of components.

2. Analyse structure of models and determine each movement pair.

3. Clarify idea of modelling.

4. Conduct modelling and test.

Using ADAMS modelling, the longitudinal velocity, lateral velocity, roll angle, pitch angle, longitudinal displacement and lateral displacement must be calculated. Dynamic characteristics of sub-components, such as push-rods, lifting system, and front and revolution system, can be accurately described by means of a multi-body dynamic analysis. As a result, the developed model, shown in Fig. 4, provides reliable dynamic performance of the platform. As one can see, each component is modelled as a rigid body connected by means of joint and/or bush to simulate platform's dynamic motion. The basic parameters used for calculation are shown in Table. I.

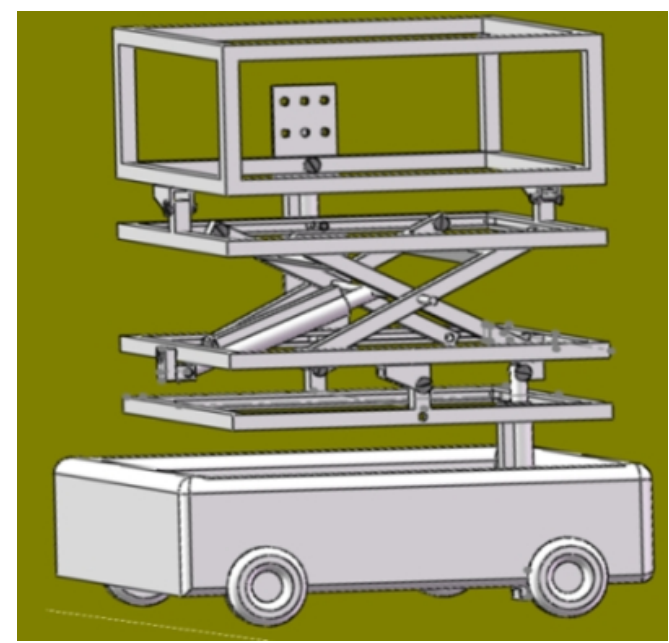

Fig. 4. ADAMS model of orchard vehicle model.

TABLE. I. BASIC PARAMETERS OF ORCHARD VEHICLE.

\begin{tabular}{|c|c|c|c|}
\hline Item & Value & Item & Value \\
\hline Width of platform & $38 \mathrm{~cm}$ & Length of platform & $38 \mathrm{~cm}$ \\
\hline Height of part A & $25 \mathrm{~cm}$ & Height of part B & $13 \mathrm{~cm}$ \\
\hline Mass of platform & $2 \mathrm{~kg}$ & Height of lifting part & $20 \mathrm{~cm}$ \\
\hline $\begin{array}{c}\text { Maximum thrust of a } \\
\text { push rod }\end{array}$ & $50 \mathrm{~N}$ & Stroke of a push rod & $100 \mathrm{~mm}$ \\
\hline $\begin{array}{c}\text { Maximum velocity of } \\
\text { a push rod }\end{array}$ & $60 \mathrm{~mm} / \mathrm{s}$ & Mass of a push rod & $0.5 \mathrm{~kg}$ \\
\hline
\end{tabular}

Co-simulation procedure can be deduced from Fig. 5 . Under ADAMS/MATLAB co-simulation, longitudinal and lateral forces due to push-rods are calculated first using MATLAB Simulink model and next implemented into ADAMS model. Whereas, longitudinal velocity, lateral velocity, roll angle, pitch angle, longitudinal displacement and lateral displacement parameters are implemented inversely from ADAMS to MATLAB Simulink model [13]-[15].

\section{B. MATLAB/Simulink Model}

The interface using S-function API (application programming interface) in ADAMS interacts with Simulink in MATLAB [16]-[18]. We select FORTRAN to create S-function and to import an adam-file, which is open in MATLAB (Fig. 6).

In accordance with the adopted control strategy (Fig. 3) and PID controller, the control system model in MATLAB /Simulink [19] has been set-up as shown in Fig. 5. The process of levelling can be divided into two independent movements along $\mathrm{X}$ and $\mathrm{Z}$ axes respectively. The Input variable of this model becomes velocity $\left(V_{X}, V_{Y}\right)$ of two push rods, corresponding to output of PID controller, as well as the angle $(\alpha, \beta)$ of the lifting platform. Since in practice, the angle variables are affected by vibrations and hardware error, an appropriate white noise was added to angle variable to make simulation results much closer to real situation.

After analysis and research test, it was found that integral coefficient $K i$ has little influence on levelling process. Therefore, $K i$ was fixed to be zero and only PD controller was used under further testing. 


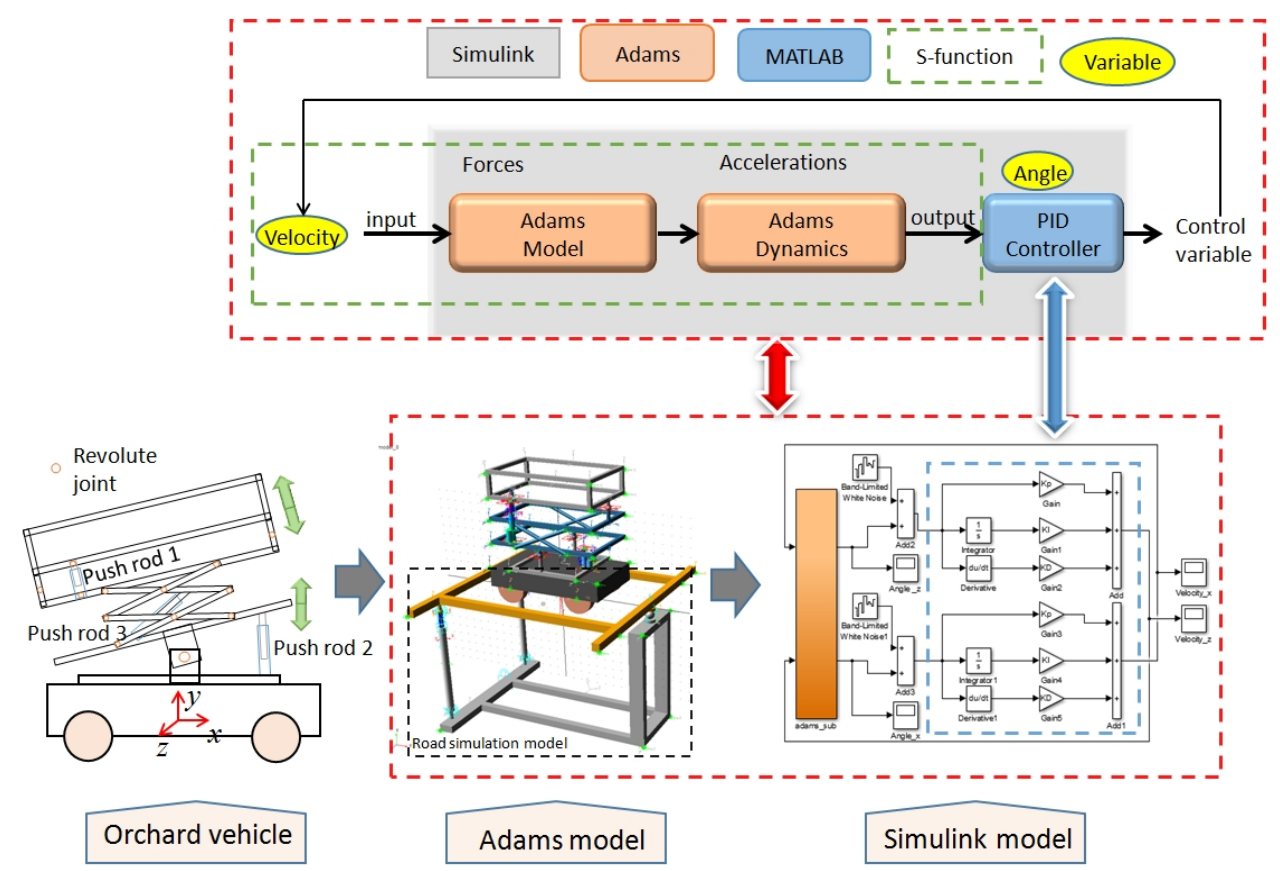

Fig. 5. Co-simulation structure of ADAMS and MATLAB Simulink.

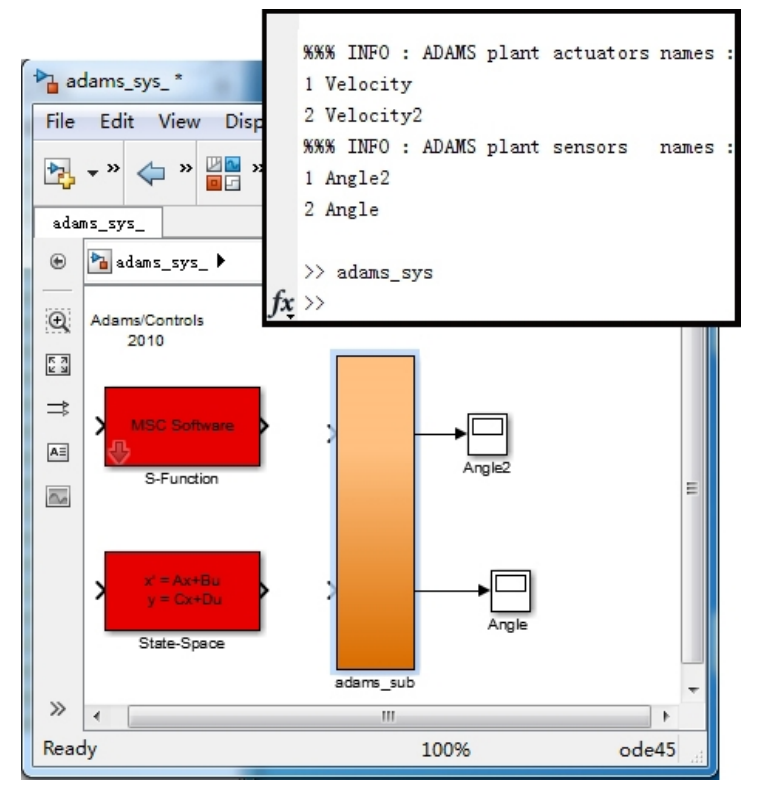

Fig. 6. Adams-file and adams_sys of Adams model in MATLAB.

\section{Simulation Result and Analysis}

It is obvious that the lifting platform motion is impacted by the road conditions. Under our consideration, the sinusoidal road was assumed to be testing road. Therefore, it was necessary to conduct respective preliminary studies to compare specific of motion of vehicle model under different road conditions. It was very helpful to set appropriate road parameters to conduct right co-simulation with ADAMS and MATLAB.

Specifically speaking, the testing vehicle model developed by means of ADAMS was used to conduct motion simulation, which was aimed to find out vehicle motion state under different road conditions. Meanwhile, the vehicle parameters (including velocity, pitching angle, pitching angular velocity, and so on) were measured and analysed using ADAMS/PostProssecor module. The longitudinal amplitude was $0.2 \mathrm{~m}$ and horizontal length was $1 \mathrm{~m}$ of each sinusoid simulation road. Its task was to examine the correct operation of control system and proper selection of values of $K p$ and $K d$ coefficients, (simulation was regarded as PID parameters tuning process) [20]. At first, the response of the control system to $K p$ variation was checked. The oscillation was found to be inevitable. However, the most convenient value of $K p$ is in the range of 20-30 (Fig. 7).

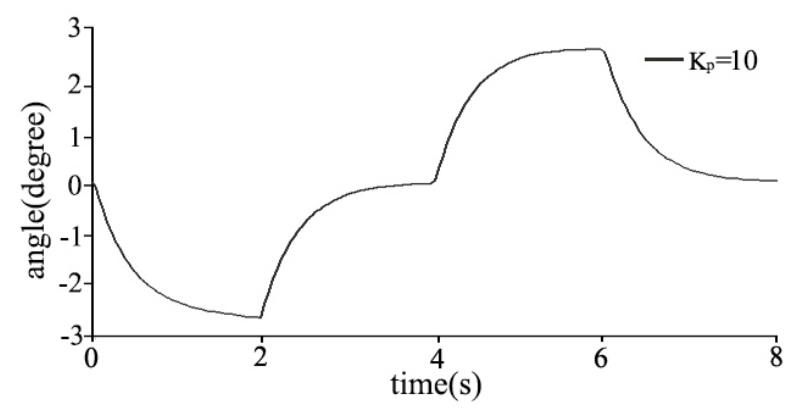

(a)

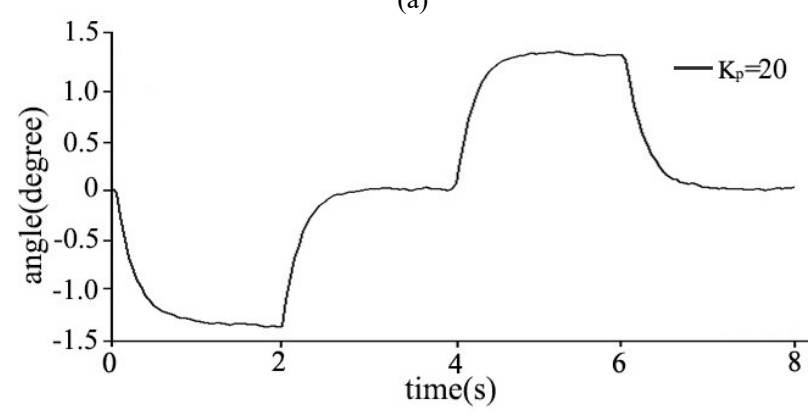

(b)

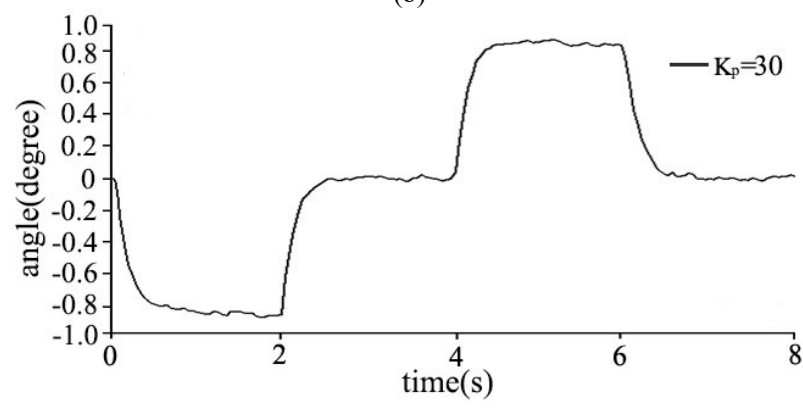

(c) 


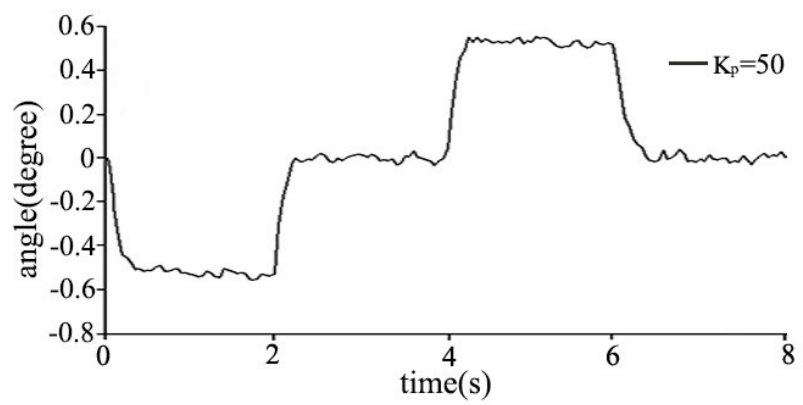

(d)

Fig. 7. Co-simulation results of $K_{p}$ optimization: (a) $-K_{p}=10, K_{d}=0$; (b) $K_{p}=20, K_{d}=0 ;(\mathrm{c})-K_{p}=30, K_{d}=0 ;(\mathrm{d})-K_{p}=50, K_{d}=0$.

The investigations have indicated a great influence of the $K p$ value on accuracy, adjustment time and oscillation level of the control system. Specifically speaking, the regulation accuracy and oscillation of platform increases as well as an adjustment time with the increase of $K p$ (Fig. 7). Therefore, in order to ensure proper operation of the system, the value of $K p$ should be limited to some extent.

According to the analysis results it can be concluded that proper selection of $K p$ can improve stability and rapidity of control system. And $K d$ value affects dynamic characteristics (Fig. 8).

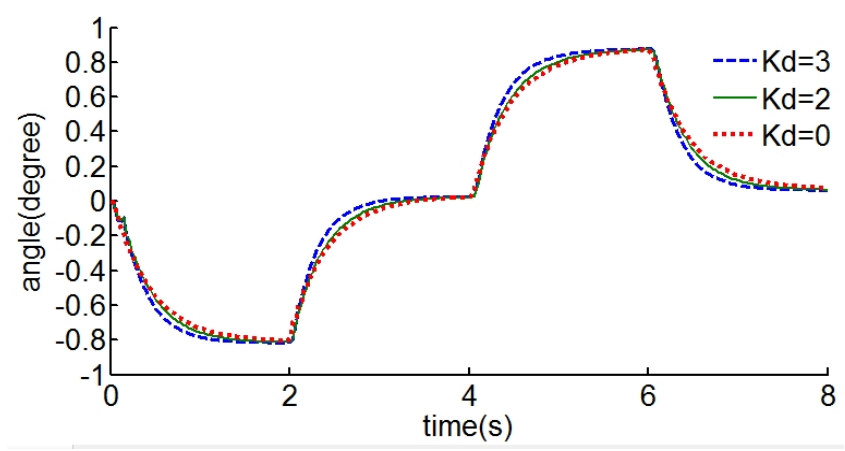

Fig. 8. Co-simulation results of $K_{d}$ optimization $\left(K_{p}=10\right)$.

For example, when value of $K d$ is equal to zero and $K p$ is very small $\left(K_{p}=10\right)$, the response is slow. But with the increase of $K d$, which from 0 to 3 , the rapidity of control system improved obviously. The performance at the initial stage is accelerated, whereas, the resultant adjustment time increases. It can be imagined that with the adjustment of $K p$ simultaneously, the stability will increase finally. Therefore, all above mentioned cases have to be taken into account under selection of the value $K d$ and $K p$

\section{EXPERIMENT RESULTS}

It is obvious that control of a full-sized vehicle under different conditions of operation is difficult to conduct in practice (unstable driven application which involves vehicle rollover and tip-over). Therefore, the testing vehicle model was established to test control algorithm, which was mentioned (Fig. 9). The developed control algorithm was able to be validated with the scaled vehicle since the lateral and vertical dynamics of testing model are similar to those of a full-sized device.

For real-time data acquisition and control, a microcontroller (STM32F103ZET6) of STM microelectronics was employed. The MPU6050 from STM which combines a 3-axis gyroscope, 3-axis accelerometer and a digital motion processor TM(DMP) were used to measure acceleration and angle rate respectively [21]-[24]. It is placed near to the C.G. of the vehicle to measure the yaw, roll, pitch angles and their rates (Fig. 10).

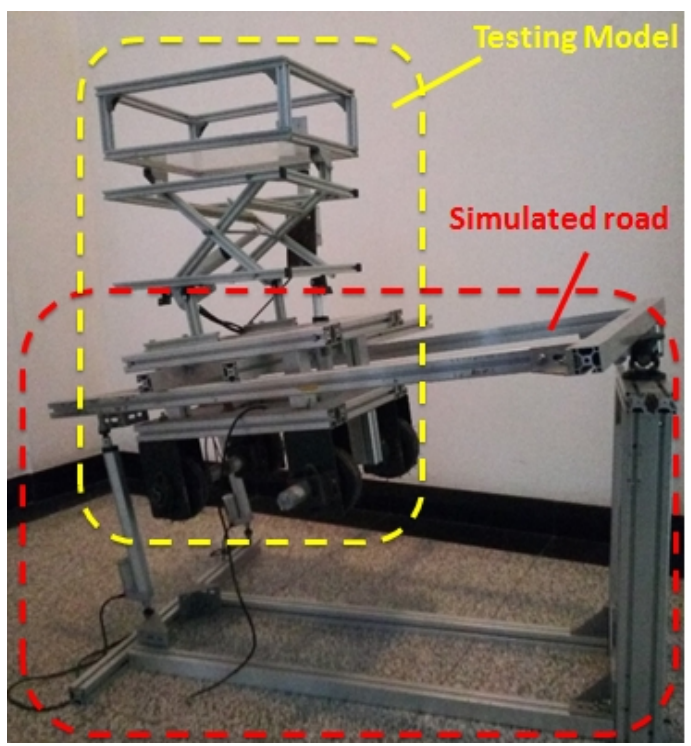

Fig. 9. Testing model and simulated road.

The push rod was driven by DC geared motor (SK3530). The microcontroller samples data from sensors and wirelessly sends them to a ZigBee receiver connected to a computer. The testing results of angle, angle velocity, acceleration and push rod speed are stored in the computer and displayed by real-time testing software, which was developed in $\mathrm{C}++$ language.

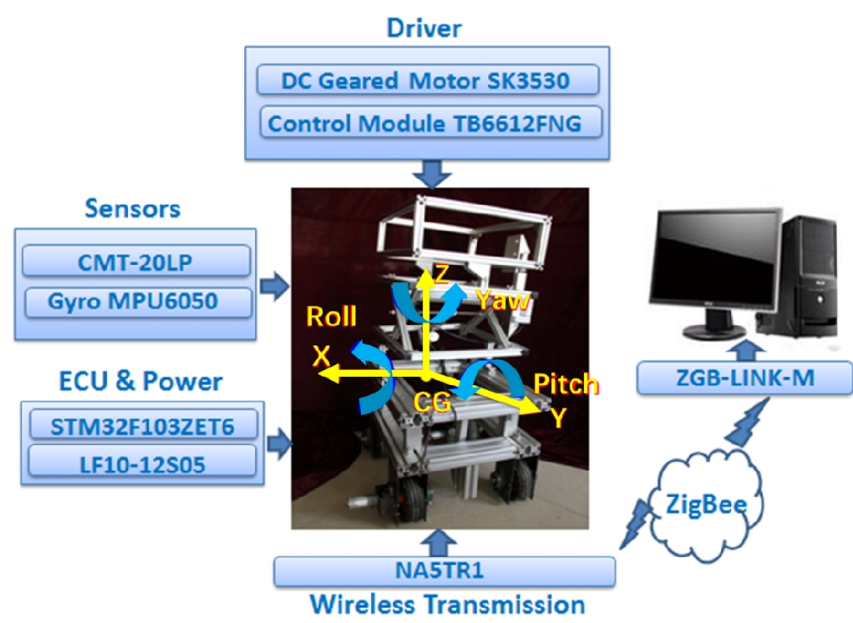

Fig. 10. Main structure of experimental system.

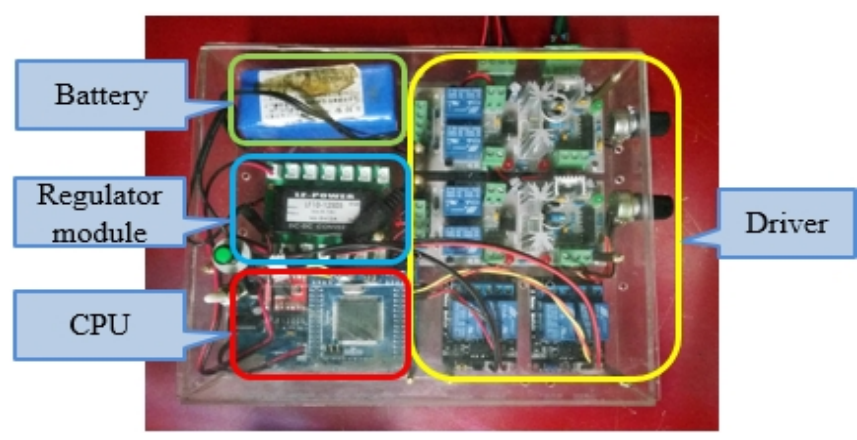

Fig. 11. Main control and driving modules. 
The main page of the testing software and its parameter setting can be seen from Fig. 12 .

Under the test, the influence of $K p$ and $K d$ value on performance of the control system was checked. It was found that simulation and test results indicate high consistency (compare Fig. 13 and Fig. 14).

For example, when $K_{p}$ value increases, within a certain range, the rapidity, accuracy, as well as stability of the control system are improved simultaneously. (For too small value of $K p$, the lifting platform becomes sluggish). On the contrary, when value of $K p$ is too high, the lifting platform movement becomes unstable. It is worth to underline that as the value of $K_{d}$ increases, the control system accelerates, however, resultant adjustment time increases.

As a result it can be concluded that the PD algorithm adopted in the control system can effectively improve the leveling process, however, with different response to adjustment of $K p$ and $K d$ parameters. From Fig. 13, with the increase of $K p$, the stability of control effect improves obviously, especially from 60 to 80 , the adjustment time is not more than $0.5 \mathrm{~s}$ and obliquity $\alpha$ of $\mathrm{X}$ axis is not more than $2.2^{\circ}$.

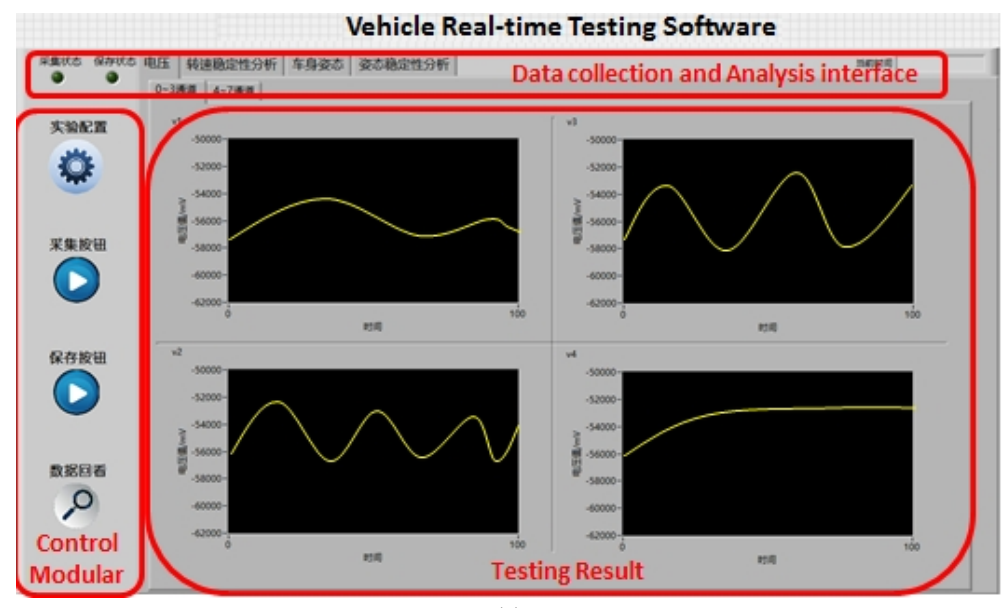

(a)

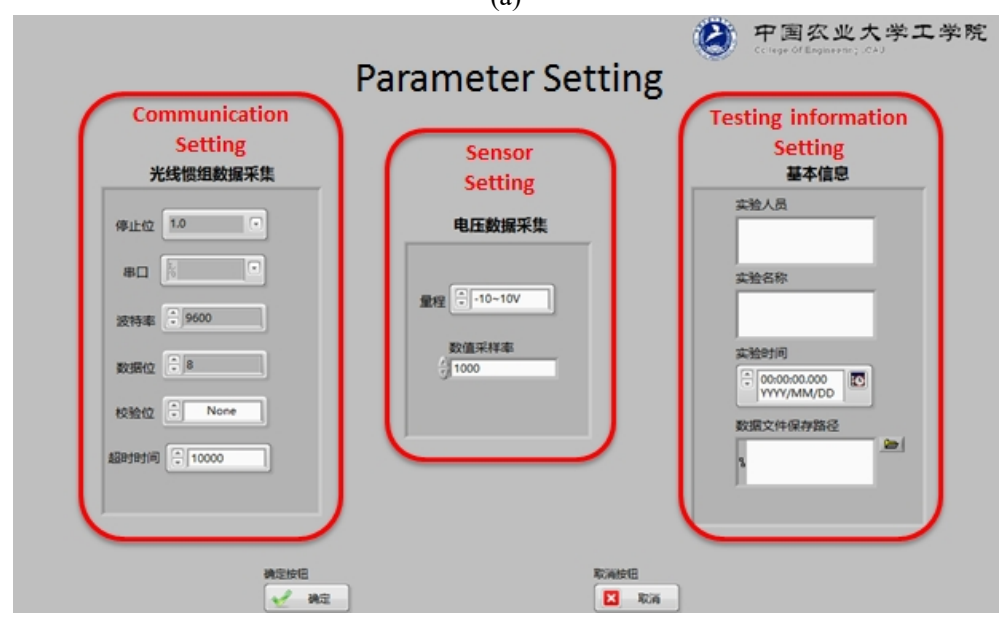

(b)

Fig. 12. Orchard vehicle real-time testing software: (a) - main page of testing software; (b) - parameter setting page.

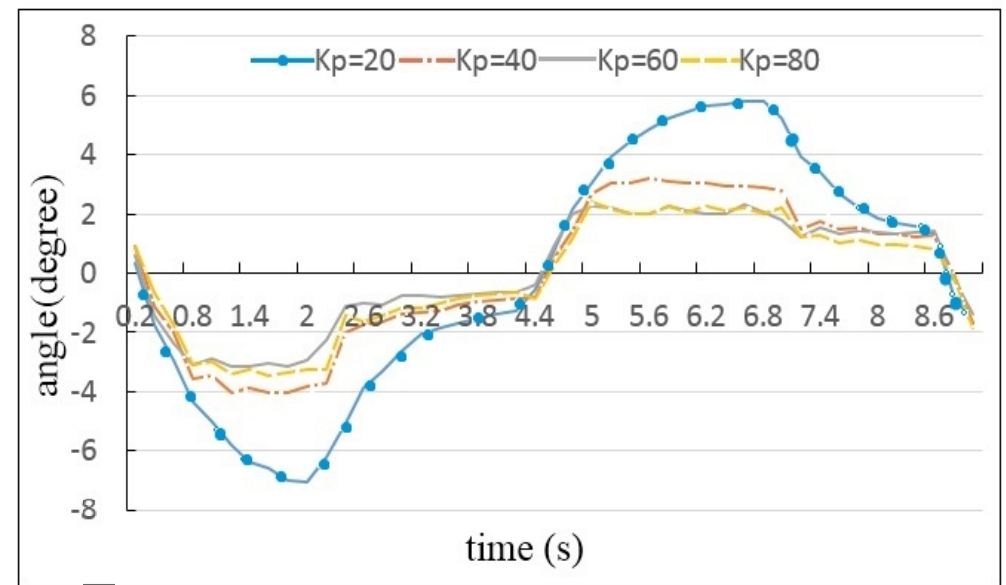

Fig. 13. Test results of $K_{p}$ vs. obliquity $\alpha$ of $\mathrm{X}$ axis $\left(K_{d}=20\right)$. 


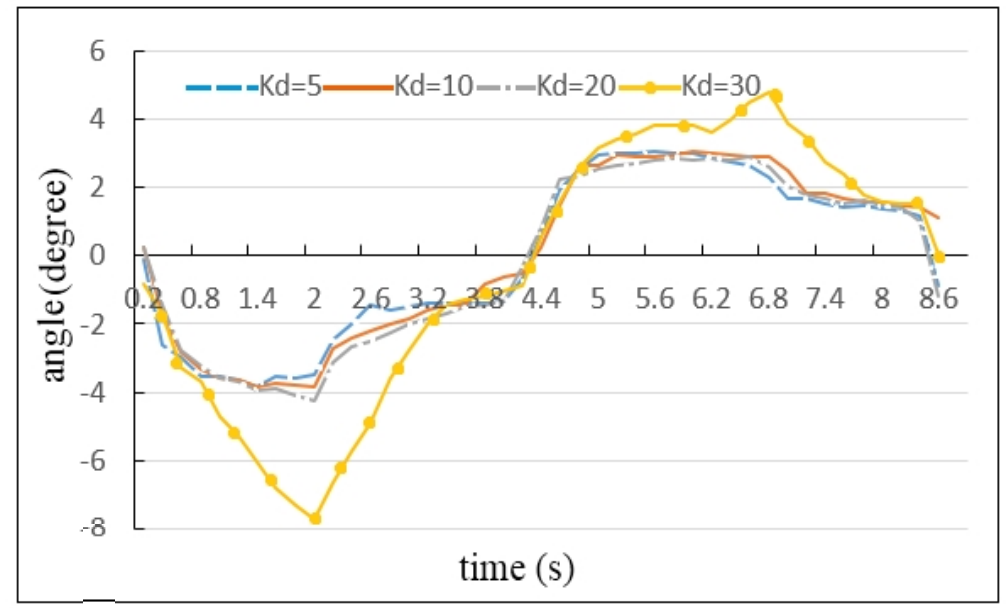

Fig. 14. Test results of $K_{d}$ vs. obliquity $\alpha$ of $\mathrm{X}$ axis $\left(K_{p}=40\right)$.

For the adjustment function of $K d$ parameter, which is shown in Fig. 14, it can be seen that, from 5 to 20, the control effects is increasing but not notable. That is mainly because of the $K_{p}=40$, which make a strong control effect of the system, with the increase of $K d$ can enhance the rapidity of control system directly. But if the $K d$ is too big (over 20), the control stability will be destroyed also. When $K_{d}=30$, the fluctuate character of balance system is too big, which cannot obtain the ideal control result.

\section{DISCUSSION}

Authors proposed a lifting platform balance control method with PD regulator control algorithm. According to attitude information of platform detected by sensors, such as angles and angular velocities, control algorithm will determine the push rods directions and calculate their velocities. The push rods then generate direct rotation around $\mathrm{X}$ and $\mathrm{Z}$ axes, which reduces angles of platform. The process is carried out cyclically until the platform achieves a balance.

Through analysis and comparison of co-simulation and vehicle model test, we can draw some interesting, particularly with practical point of view, conclusions. When $K_{p}$ value increases, the rapidity, accuracy, and stability of the control system can be improved significantly. But when it is over a certain value (it is 50 in simulation), the lifting platform becomes sluggish or unstable. As for $K d$, with its increase, response of system accelerates, whereas the total adjustment time increases. Besides, satisfactory performance can be achieved when combine the control algorithm with properly selected PD regulator parameters.

\section{CONCLUSIONS}

1. Stability of control of the lifting platform has been verified basing on its original structure, designed by authors.

2. The PD regulator control algorithm is proposed to compensate for errors of unbalance angles. Co-simulation and model test are conducted to verify the control algorithm.

3. In co-simulation, the angles of platform are controlled to small values by adjustment of PD parameters. Obviously, the algorithm can realize balance control of platform. The scale model test is carried out to verify the control method in a more practical condition. The balance control can also be achieved though it is not as good as that in simulation.

As a result, the stability control algorithm developed and recommended in this paper offers an additional improvement for lifting platform production and can be also helpful under solving related aspects.

\section{REFERENCES}

[1] J. Chao, X. C. Zhu, B. Yao, G. L. Tao, Q. F. Wang, "Levelling control of an electro-pneumatic stabilized platform driven by pneumatic muscles", in Proc. 6th IFAC Symposium on Mechatronic Systems. IFAC Volumes, Hangzhou, China, 2013, pp. 445-452. [Online]. Available: https://doi.org/10.3182/20130410-3-CN-2034.00083

[2] J. Zhang, D. Huang, C. Lu, "Research on dynamic model and control strategy of auto-levelling system for vehicle-borne platform", Int. Conf. Mechatronics and Automation, Piscataway, NJ, USA, 2007. [Online]. Available: https://doi.org/10.1109/ICMA.2007.4303679

[3] O. Krejcar, "Problem Solving of Low Data Throughput on Mobile Devices by Artefacts Prebuffering", EURASIP Journal on Wireless Communications and Networking. vol. 2009, article ID 802523, pp. 1-8. [Online]. Available: http://dx.doi.org/10.1155/2009/802523

[4] T. S. Hong, M. Ghobakhloo, W. Khaksar, "Robotic welding technology", Comprehensive Materials Processing, vol. 6, pp. 77-99, 2014. [Online]. Available: http://dx.doi.org/10.1016/B978-0-08096532-1.00604-X

[5] H. Gattringer, R. Riepl, M. Neubauer, "Optimizing industrial robots for accurate high-speed applications", Journal of Industrial Engineering, 2013. [Online]. Available: http://dx.doi.org/ $10.1155 / 2013 / 625638$

[6] M. I. Ullah, S. A. Ajwad, R. U. Islam, U. Iqbal, J. Iqbal, "Modeling and computed torque control of a 6 Degree of Freedom robotic arm", in Proc. IEEE Int. Conf. on Robotics and Emerging Allied Technologies in Engineering, Islamabad, Pakistan, 2014, pp. 133-138. [Online]. Available: http://dx.doi.org/10.1109/icreate.2014. 6828353

[7] Y. Singh, V. Vinoth, M. Santhakumar, "Dynamic modelling and control of a 3-DOF planar parallel robotic (XY $\theta \mathrm{z}$ motion) platform", Procedia Materials Science, vol. 5, pp. 1528-1539, 2014. [Online]. Available: https://doi.org/10.1016/j.mspro.2014.07.340

[8] J. W. Yue, Z. T. Zhou, J. Jiang, Y. D. Liu, D. W. Hu, "Balancing a simulated inverted pendulum through motor imagery: An EEG-based real-time control paradigm", Neuroscience Letters, vol. 524, no. 2, pp. $95-100,2012$. [Online]. Available: https://doi.org/10.1016/ j.neulet.2012.07.031

[9] J. Cao, X. C. Zhu, B. Yao, G. L. Tao, Q. F. Wang, "Levelling control of an electro-pneumatic stabilized platform driven by pneumatic muscles", IFAC Proc. Volumes, vol. 46, no. 5, pp. 445-452, 2013. [Online]. Available: https://doi.org/10.3182/20130410-3-CN-2034. 00083

[10] J. Lin, S. Y. Guo, J. L. Chang, "Fuzzy coordinator compensation for balancing control of cart-seesaw system", Journal of Sound and Vibration, vol. 330, no. 26, pp. 6296-6310, 2011. [Online]. Available: https://doi.org/10.1016/j.jsv.2011.08.003 
[11] O. S. Bhatti, K. Mehmood-ul-Hasan, M. A. Imtiaz, "Attitude control and stabilization of a two-wheeled self-balancing robot", Journal of Control Engineering and Applied Informatics, vol. 17, no. 3, pp. 98-104, 2015.

[12] A. Sapietova, V. Dekys, "Dynamic analysis of rotating machines in MSC.ADAMS", Procedia Engineering, no. 136, pp. 143-149, 2016 [Online]. Available: https://doi.org/10.1016/j.proeng.2016.01.188

[13] P. Q. Li, J. He, "Geometric design safety estimation based on tire-road side friction", Transportation Research Part C: Emerging Technologies, no. 63, pp. 114-125, 2016. [Online]. Available: https://doi.org/10.1016/j.trc.2015.12.009

[14] S. Janani, "Design of integral constant state feedback controller using Ackermann's function", IOSR Journal of Electronics and Communication Engineering, vol. 9, no. 1, pp. 58-63, 2014. [Online]. Available: http://dx.doi.org/10.9790/2834-09115863

[15] K. Ibrahim, A. Ramadan, M. Fanni, Y. Kobayashi, A. Abo-Ismail, M. G. Fujie, "Development of a new 4-DOF endoscopic parallel manipulator based on screw theory for laparoscopic surgery", Mechatronics, vol. 28, pp. 4-17, 2015. [Online]. Available: https://doi.org/10.1016/j.mechatronics.2015.02.006

[16] A. Namadmalan, J. S. Moghani, "Single-phase current source induction heater with improved efficiency and package size", Journal of Power Electronics, vol. 13, no. 2, pp. 322-328, 2013. [Online]. Available: http://dx.doi.org/10.6113/JPE.2013.13.2.322

[17] S. Lisauskas, R. Rinkeviciene, "Model of ventilator drive with fuzzy controller", in Proc. of the $20^{\text {th }}$ Int. Conf. Electromagnetic disturbances, Kaunas, 2010. pp. 107-110.

[18] X. Wang, X. Miao, W. W. Li, H. S. Lu, "Study on orchard vehicle motion stability control system", IFAC-PapersOnLine, vol. 49, no. 6 , pp. 451-456, 2016. [Online]. Available: https://doi.org/10.1016/ j.ifacol.2016.10.082

[19] D. Sun, D. Chen, S. M. Wang, X. Wang, "A dynamic instability detection and prediction system for high clearance tractor", IFAC-Papers Online, vol. 49, no. 16, pp. 50-54, 2016. [Online] Available: https://doi.org/10.1016/j.ifacol.2016.10.010

[20] J. Guo, H. Jafarkhani, "Sensor deployment with limited communication range in homogeneous and heterogeneous wireless sensor networks", IEEE Trans. Wireless Communications, vol. 15, no. 10, pp. 6771-6784, 2016. [Online]. Available: https://doi.org/ 10.1109/TWC.2016.2590541

[21] S. Borekci, S. Oncu, "Switching-mode BJT driver for self-oscillated push-pull inverters", Journal of Power Electronics, vol. 12, no. 2, pp. 242-248, 2012. [Online]. Available: http://dx.doi.org/10.6113/JPE. 2012.12.2.242

[22] X. Wang, S. Q. Zhao, L. Y. Wang, S. B. Xu, "Stability control method fororchard vehicle", Nongye Gongcheng Xuebao/transactions of the Chinese Society of Agricultural Engineering, vol. 46, no. 5, pp. 13-19, 2015.

[23] R. Kalaivani, P. Lakshmi, "Biogeography-based optimization of PID tuning parameters for the vibration control of active suspension system", Journal of Control Engineering and Applied Informatics, vol. 16, no. 1, pp. 31-39, 2014. 